\title{
Cardiolipin: Setting the beat of apoptosis
}

\author{
François Gonzalvez • Eyal Gottlieb
}

Published online: 6 February 2007

C Springer Science + Business Media, LLC 2007

\begin{abstract}
Cardiolipin (CL) is a mitochondria-specific phospholipid which is known to be intimately linked with the mitochondrial bioenergetic machinery. Accumulating evidence now suggests that this unique lipid also has active roles in several of the mitochondria-dependant steps of apoptosis. $\mathrm{CL}$ is closely associated with cytochrome $c$ at the outer leaflet of the mitochondrial inner membrane. This interaction makes the process of cytochrome $c$ release from mitochondria more complex than previously assumed, requiring more than pore formation in the mitochondrial outer membrane. While CL peroxidation could be crucial for enabling cytochrome $c$ dissociation from the mitochondrial inner membrane, cytochrome $c$ itself catalyzes CL peroxidation. Moreover, peroxy-CL directly activates the release of cytochrome $c$ and other apoptogenic factors from the mitochondria. CL is also directly involved in mitochondrial outer membrane permeabilization by enabling docking and activation of proapoptotic Bcl-2 proteins. It appears therefore that CL has multiple roles in apoptosis and that CL metabolism contributes to the complexity of the apoptotic process.
\end{abstract}

Keywords Mitochondria $\cdot$ Apoptosis · Cardiolipin

\section{Background}

It has long been known that mitochondria are the ATP generating powerhouse of the cell and the site of other key metabolic pathways involving fatty acid, amino acid and steroid metabolism. However, in the early 1990s it became

F. Gonzalvez · E. Gottlieb $(\square)$

Cancer Research UK, The Beatson Institute for Cancer Research, Glasgow, G61 1BD, United Kingdom

e-mail: e.gottlieb@beatson.gla.ac.uk clear that in addition to these critical life-supporting roles, mitochondria play a central part in the execution of apoptotic cell death.

The involvement of mitochondria in apoptosis first came into focus with the discoveries that most pro-apoptotic stimuli induce an early release of mitochondrial proteins, which activates the cellular apoptotic program and disrupts mitochondrial bioenergetics $[1,2]$. These mitochondrial proteins, known as apoptogenic factors, include cytochrome $c$, smac/diablo, HtrA2/Omi, AIF and endonuclease G, of which cytochrome $c$ has been the most intensively studied. Cytochrome $c$ is normally involved in the ATP generation pathway in mitochondria, transferring electrons from the cytochrome bc1 complex (complex III) to cytochrome oxidase (complex IV). But once released into the cytosol, cytochrome $c$ induces a cytochrome c/dATP/Apaf-1/pro-caspase-9 complex termed the apoptosome [3]. The apoptosome activates caspase-3, resulting in the degradation of many cellular components. Cells deficient in cytochrome $c$, Apaf-1, caspase-9 or caspase-3 have impaired apoptosis in response to intrinsic mitochondria-dependent signals, underpinning the importance of these components [4-7].

In response to apoptotic signals coming from the cytosol, mitochondria-dependent apoptosis requires permeabilization of the mitochondrial outer membrane. Mitochondrial membrane permeabilization is tightly regulated by proteins of the Bcl-2 family and is often considered the point of no return in the apoptotic signalling cascade, leading to several events such as DNA degradation in the nucleus and exposure of phosphatidylserine (PS) on the outer leaflet of the plasma membrane $[8,9]$.

PS was the first lipid identified to have a role in apoptosis regulation, when it was shown that exposure of PS on the surface of apoptotic lymphocytes forms a recognition site for phagocytosis by macrophages [10]. The sphingolipid 


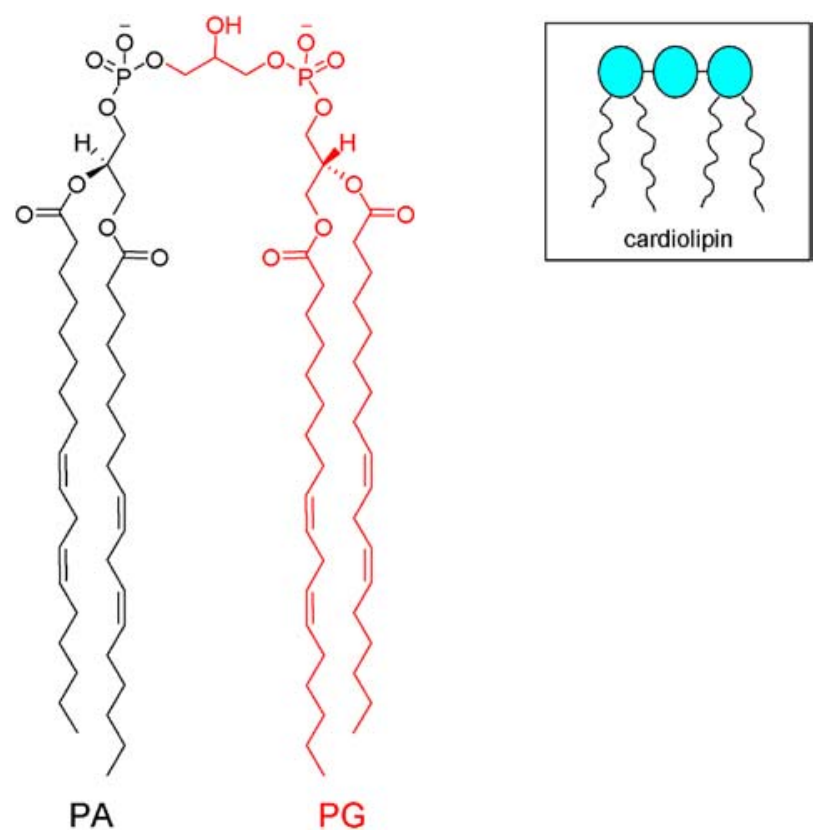

Fig. 1 Molecular (left) and schematic (inset) structure of cardiolipin. Cardiolipin is a dimer of phosphatidylglycerol (PG) and phosphatidic acid (PA). It comprises four acyl chains, two phosphate groups and three glycerols (schematically represented by blue circles). Under physiological $\mathrm{pH}$ one of the phosphate groups is de-protonated, making cardiolipin a negatively-charged phospholipid

ceramide has attracted much attention in recent years. Cellular ceramide levels increase in response to a wide variety of apoptotic stimuli (e.g. TNF $\alpha$, Fas ligand, IFN $\gamma$, staurosporine and etoposide) preceding the mitochondrial steps of apoptosis [11]. Although the mechanism of ceramidemediated apoptosis is still a matter of debate, a growing body of evidence supports a direct effect of ceramide on mitochondria resulting in alterations in bioenergetics, generation of reactive oxygen species and permeabilization of the mitochondrial outer membrane [11].

Another class of lipids termed cardiolipin (CL) has attracted new interest in the field of cell death. CL is a glycerolbased phospholipid (Fig. 1) most of which is found in the mitochondrial inner membrane. Several independent studies suggest that CL has either survival- or death-supporting roles in cells. This review summarizes recent data and discusses the complex and controversial role of CL in apoptosis.

\section{Cardiolipin physiology}

\section{Cardiolipin synthesis}

The name "cardiolipin" alludes to the fact that it was first isolated from bovine heart. CL or diphosphatidylglycerol, whose dimeric structure distinguishes it from other glycerophospholipids, has the glycerol-phosphate and two acyl groups of each monomer bound together through a single glycerol head. This results in four acyl chains, three glycerols and two phosphate groups per molecule (Fig. 1). CL is detected exclusively in bioenergetic membranes such as those of bacteria and mitochondria, thus providing more evidence for the endosymbiotic origin of mitochondria. The biosynthetic pathway of CL in mammals has been well described [12,13]. Briefly, CL is synthesized de novo in a four step pathway catalyzed by four mitochondrial enzymes, yielding a $\mathrm{CL}$ archetype. The first three steps correspond to the phosphatidylglycerol (PG) pathway and pass trough the generation of the common intermediates, phosphatidic acid (PA), cytidine-5'-diphosphate-diacylglycerol (CDP-DG) and phosphatidylcytidine-monophosphate (CMP). The final step is unique to CL synthesis and is catalyzed by CL synthase. In this reaction, a molecule of PG condenses with a molecule of the intermediate CDP-DG to yield diphosphatidylglycerol or CL. The human CL synthase gene has recently been identified by its ability to restore a CL profile to the CL synthase deficient yeast mutant $c r d l \Delta$ [14]. Considering the variety of fatty acids, the number of potential combinations of the acyl chains is high, and indeed, the pattern of CL molecular species varies between organisms and even between tissues. Eukaryotic CL has a characteristic acyl chain pattern which is restricted to 18 carbons [15]. In human heart the predominant $\mathrm{C}_{18}$ fatty acid is linoleic acid $\left(\mathrm{C}_{18: 2}\right)$ so heart $\mathrm{CL}$ contains mostly $\mathrm{C}_{18: 2}$ acyl chains. However human lymphoblast $\mathrm{CL}$ contains predominantly oleyl chains $\left(\mathrm{C}_{18: 1}\right)$ [16]. The significance of this specificity is still not understood. Interestingly, the enzymes involved in the pathway of CL synthesis exhibit no selectivity for a specific acyl chain length [17]. Therefore, once synthesized in mitochondria, a maturation step replacing the original acyl chains with specific $\mathrm{C}_{18}$ unsaturated ones is required.

The generation of mature CL requires a cycle of two reactions: the hydrolysis of one original acyl chain to generate a monolyso-CL (MLCL), now containing only three acyl groups, followed by the reacylation of MLCL with a specific $\mathrm{C}_{18}$ acyl chain. Phospholipase A2 catalyzes the first step of acyl chain removal [18]. However, the enzyme catalyzing MLCL-acyltransferase activity has not been identified [18, 19].

Barth syndrome (OMIM \# 302060) is the only human genetic disorder discovered where alterations of CL metabolism are a primary cause of disease [20, 21]. This $\mathrm{X}$-linked genetic disorder is due to mutations in the tafazzin gene (TAZ) located on region Xq28 [22]. Analyses of the CL profile of different tissues obtained from Barth syndrome patients revealed a decrease in CL and an increase in MLCL and sequence alignments of $T A Z$ showed homology with the glycerolipid acyltransferase family [23-25]. While these data suggest TAZ encodes the mitochondrial MLCL acyltransferase, the biochemical function of Tafazzin has not been fully characterized. 


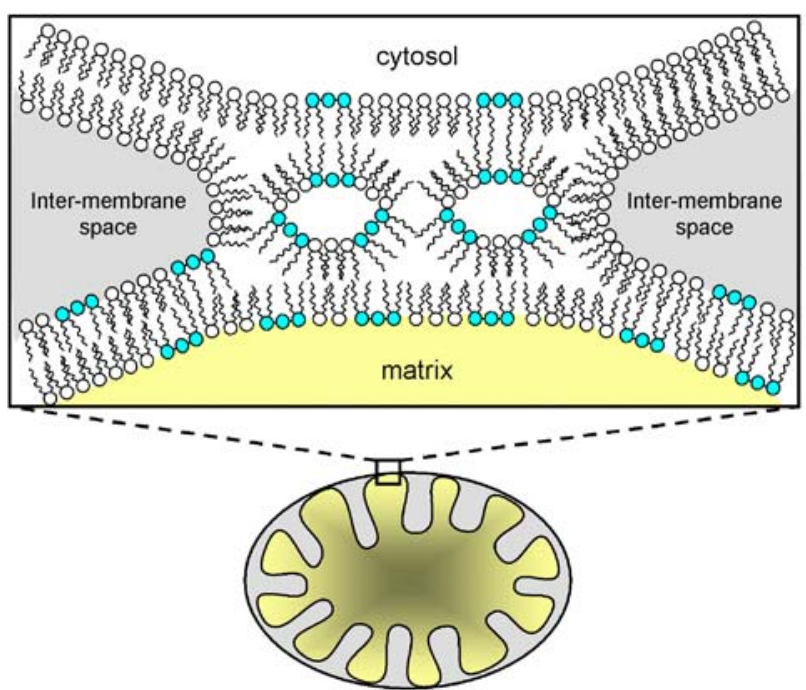

Fig. 2 The non-bilayer hexagonal structure of lipids was characterised in vitro and proposed to contribute to the structure of contact sites between the inner and outer membranes of mitochondria. Negativelycharged phospholipids (such as cardiolipin) are more likely to adopt this structure which may fuse the mitochondrial membranes at the contact sites and redistribute cardiolipin on the cytosolic face of the mitochondria

\section{Cardiolipin localization in mitochondria}

$\mathrm{CL}$ is specific to mitochondrial membranes but its precise location within the different compartments of the organelle is still the subject of controversy. For many years CL was assumed to be associated exclusively with the mitochondrial inner membrane where, as measured in bovine heart mitochondria, it represents $\sim 25 \%$ of the total phospholipids [26]. More recently CL has also been identified in the mitochondrial outer membrane $(\sim 4 \%)$ and especially at the contact sites connecting the outer membrane with the inner one [27-29]. Through the contact sites, CL may reach the mitochondrial outer membrane and the cytosolic face of the mitochondria. This notion is supported by the fact that the two major phospholipids present in contact sites, phosphatidylethanolamine (PE) and CL ( $25 \%$ each) have the ability to adopt a non-bilayer hexagonal $\mathrm{H}_{\mathrm{II}}$ phase in vitro (Fig. 2) [30]. Such structures can contribute to the fusion of two membranes [31].

The role of cardiolipin in bioenergetics

The exclusive presence of CL in bioenergetic membranes suggests that it interacts with the electron transport chain complexes involved in oxidative phosphorylation. Indeed, CL is required for optimal activity of complex I (NADH:ubiquinone oxido-reductase), complex III (ubiquinone:cytochrome $c$ oxido-reductase), complex IV (cytochrome $c$ oxidase) and complex V (ATP synthase), four large complexes integrated in the inner mitochondrial membrane [32, 33]. Further, complexes III, IV and V were shown to contain $\mathrm{CL}$ in their quaternary structure [32, 34, 35] and CL was observed within the 3D crystal structure of Eschericia Coli succinate dehydrogenase, an ortholog of the mitochondrial respiratory complex II (succinate:ubiquinone oxido-reductase) [36]. CL is also required by mitochondrial substrate carriers, including the adenine nucleotide translocator (ANT), acylcarnitine translocase and phosphate carrier [37-40]. It was reasonable to predict therefore that a deficiency in CL would result in alterations in cell respiration. A Chinese hamster ovary $(\mathrm{CHO})$ cell line containing a temperature-sensitive $(t s)$ mutant of $\mathrm{PG}$ synthase (CHO-PGS-S) has provided the first indication of the potential involvement of $\mathrm{CL}$ in cellular bioenergetics [41]. At the non-permissive temperature $\left(40^{\circ} \mathrm{C}\right)$ these cells exhibit a decrease in oxygen consumption and ATP production, accompanied by a compensatory increase in glycolysis [42]. However, since these cells have reduced levels of both PG and $\mathrm{CL}$ at $40^{\circ} \mathrm{C}$, it is not possible to attribute these bioenergetic defects to CL alone. Other studies using the CL synthase deficient yeast mutant $\operatorname{crdl} \Delta$, have provided more direct evidence for the requirement of CL for mitochondrial bioenergetics. Somewhat surprisingly, the $\operatorname{crdl} \Delta$ mutant could grow, though not as efficiently as wild type yeast, on non-fermentable carbon sources, indicating that $\mathrm{CL}$ is not essential for oxidative phosphorylation [43]. However, several bioenergetic defects, associated with a reduction of ANT activity, reduced mitochondrial membrane potential and an overall decrease in oxidative phosphorylation, were observed in the crdl $\Delta$ mutant when grown under stress conditions [44-46]. Thus, CL appears to be required for sustained mitochondrial inner membrane integrity and function.

Cytochrome $c$ is an essential hemoprotein which functions as a mobile electron carrier between complex III and complex IV. Only $15 \%$ of cytochrome $c$ is free in the intermembrane space $[47,48]$ while most of it is attached to the mitochondrial inner membrane via specific interactions with CL [49, 50]. Two types of interactions, hydrophobic and electrostatic, have been linked to two distinct CL binding sites on cytochrome $c$. Initially, these interactions were thought to play a role in the electron-shuttling activity of cytochrome $c$ by keeping the molecule in the proximity of the respiratory chain [50]. More recently, CL-cytochrome $c$ interactions were suggested to participate in the regulation of apoptosis (see below).

Cardiolipin maintains the structure of the mitochondrial inner membrane

The mitochondria of CHO-PGS-S cells appear swollen and have disorganized cristae [42, 51]. However, as mentioned above, these alterations cannot be solely attributed to $\mathrm{CL}$ since these cells lack both CL and PG. A more recent study 
of HeLa cells in which the expression of CL synthase was decreased by RNA interference (RNAi) indicated that CL is directly required for maintenance of mitochondrial structure [52]. This report, however, contrasts with the phenotype of the $\operatorname{crdl} \Delta$ yeast mutants, which lack CL but maintain normal mitochondrial morphology [53]. The differences between CL synthase-deficient mammalian cells and yeast may be due the ability of PG to supplant the membrane-preserving function of $\mathrm{CL}$ in yeast.

\section{Cardiolipin in relation to apoptosis}

Cardiolipin levels and oxidative stress

Loss of CL is associated with diverse pathophysiological conditions such as ageing and ischemia/reperfusion processes $[54,55]$. For example, the loss of CL during ischemia/reperfusion is followed by a decrease in oxidative phosphorylation which may contribute to myocyte death in the peri-infarct regions of the ischemic myocardium. The decline in mitochondrial respiratory functions the accumulation of reactive oxygen species (ROS). Under normal physiological conditions mitochondrial CL may protect cells from oxidative stress in part through the deacylation-reacylation cycle discussed above. However, CL is also a vulnerable target of ROS due to its unsaturated acyl chains and its close proximity to ROS generation sites. ROS cause the peroxidation of $\mathrm{CL}$ and a parallel decrease in the activities of complexes I and IV [56, 57]. Currently this seems to be very much a "chicken and egg" issue, and it is unclear whether ROS trigger the loss of CL or whether loss of CL triggers ROS generation. It is clear, however, that during many cell death processes ROS and loss of CL are closely linked in a cycle of CL peroxidation. Peroxidation of CL also occurs following a variety of apoptotic stimuli such as nitric oxide, Fas receptor stimulation, NGF deprivation, staurosporine and actinomycin D [58-60]. Interestingly, apoptosis via a pathway involving a decrease in CL synthesis was seen in neonatal rat cardiac myocytes and in breast cancer cells treated with saturated fatty acids, particularly palmitate $[61,62]$.

Cardiolipin-Cytochrome $c$ interactions regulate cytochrome $c$ release

As mentioned above, the majority of cytochrome $c$ is bound to the outer leaflet of the mitochondrial inner membrane. Cytochrome $c$ has a net charge of +8 at physiological $\mathrm{pH}$ allowing it to bind membranes primarily through electrostatic interactions with the head groups of anionic phospholipids such CL $[49,50]$. Cytochrome $c$ has a hydrophobic cavity which may account for hydrophobic interactions with the fatty acyl chains of CL [63]. Two CL binding sites on cytochrome $c$ have been proposed; the A-site which facilitates electrostatic interactions with the negative charges of $C L$ and the $\mathrm{C}$-site which is involved in hydrophobic interactions with the fatty acyl chain of CL [50]. These sites are responsible for two different conformations of cytochrome $c$ in the intermembrane space: a loosely bound conformation involving site $\mathrm{A}$ and a tightly bound conformation at site $\mathrm{C}$ that partially embeds the protein in the membrane [64]. Loosely bound cytochrome $c$ participates in the transfer of electrons from complex III to complex IV, as well as in ROS scavenging $[65,66]$. Tightly bound cytochrome $c$ was proposed to possess peroxidase activity that utilizes hydrogen peroxide generated in the mitochondria to peroxidate CL (see below) [58].

For both types of CL binding it was proposed that cytochrome $c$ release from mitochondria would first require the dissociation of its interactions with CL (Fig. 3) [67, 68]. This is consistent with recent findings showing that in CL-deficient cells, a greater fraction of cytochrome $c$ is free or loosely bound [52]. The fact that, in vitro, cytochrome $c$ has a lower affinity for peroxidized CL than CL, suggests that CL peroxidation may enable cytochrome $c$ detachment from the inner membrane (Fig. 3). Complete release of cytochrome $c$ into the inter-membrane space requires dissociation of both the hydrophobic and the electrostatic interactions between cytochrome $c$ and CL [69]. The final release of cytochrome $c$ from mitochondria requires additional steps in the process, consisting of the permeabilization of the outer membrane. Cristae remodelling was also shown to be required for cytochrome $c$ re-distribution within the mitochondrial inter-membrane space before its release [70] but whether the dissociation of CL-cytochrome $c$ interactions is related to this process awaits further studies. Still, the studies described above strongly indicate that CL and cytochrome $c$ are physically associated and for some functions at least they are also interdependent.

\section{Cardiolipin: Docking site for tBid}

Bid is a pro-apoptotic protein of the diverse Bcl-2 family, possessing sequence homology only within the conserved Bcl-2 Homology 3 (BH3) domain [71]. Bid has attracted increasing interest since it was identified to be a substrate of caspase-8 in response to activation of death receptors such as Fas. During apoptosis, N-terminal cleavage of Bid by caspase- 8 produces $\mathrm{p} 15 \mathrm{tBid}$, the active form which rapidly targets mitochondria and triggers cytochrome $c$ release [72, 73]. One important target of tBid on the mitochondrial outer membrane is Bax, a multi-BH domain pro-apoptotic Bcl-2 protein which interacts with the $\mathrm{BH} 3$ domain of tBid [74]. In fact, tBid binding to the first $\alpha$ helix of Bax was shown to be crucial for the pro-apoptotic activity of tBid [74]. 
Fig. 3 Cytochrome $c$ (red) is attached to cardiolipin on the outer surface of the mitochondrial inner membrane and therefore, permeabilization of the mitochondrial outer membrane is not sufficient for cytochrome $c$ release. The dissociation of cytochrome $c$ from cardiolipin is a required step prior to outer membrane permeabilization and is triggered by cardiolipin peroxidation. Recently it was shown that cardiolipin peroxidation is catalyzed by the bound cytochrome $c$ itself [58]
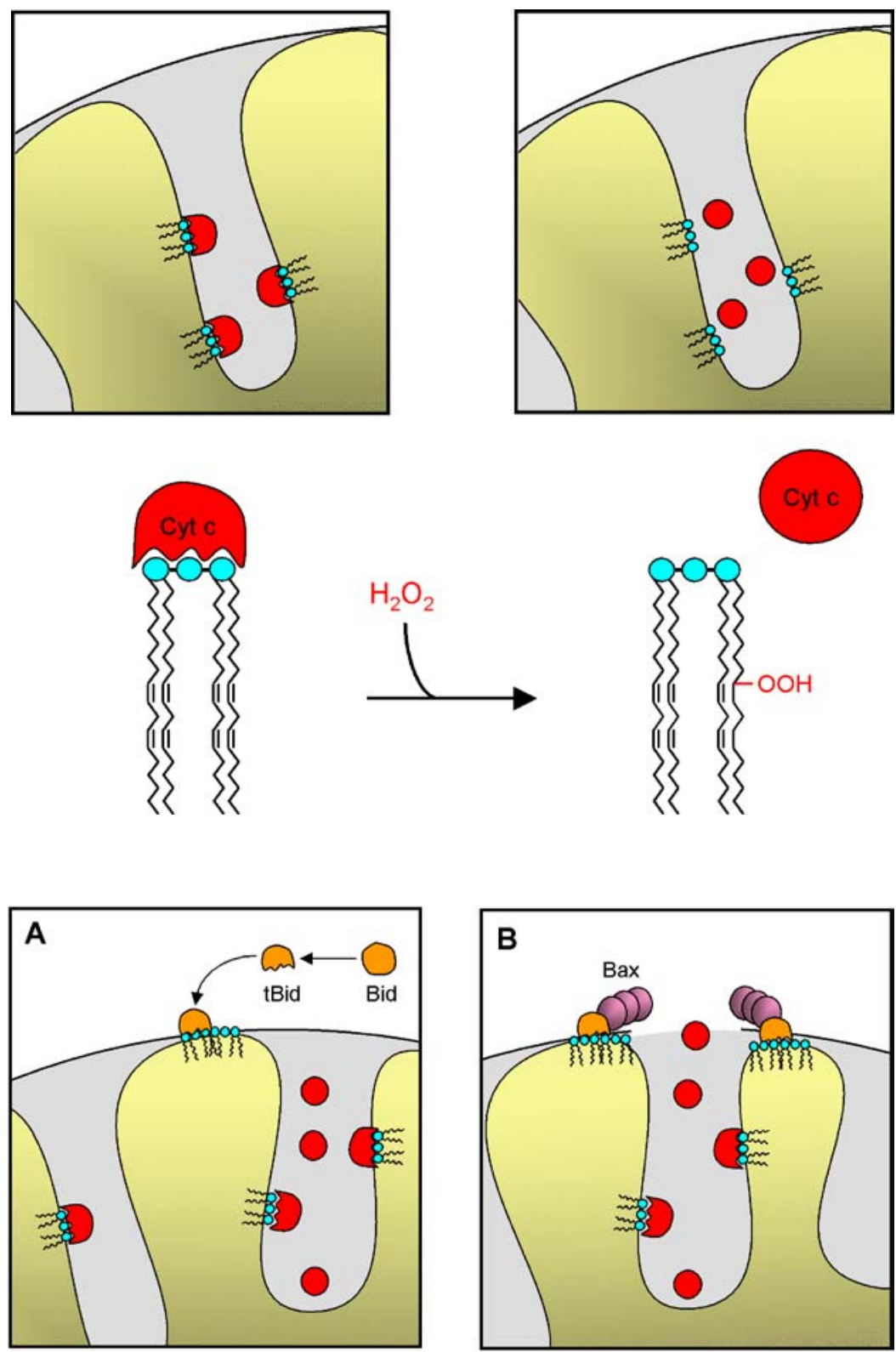

Fig. 4 Cardiolipin executes apoptosis-supporting roles at the mitochondrial outer membrane. (A) Cardiolipin serves as a docking platform for the pro-apoptotic $\mathrm{Bcl}-2$ protein tBid, particularly at contact sites of the inner and outer membranes. (B) Cardiolipin assists the perforation of the mitochondrial outer membrane by tBid and Bax. The mechanism is still elusive
The first apoptosis-promoting role of CL emerged from biochemical studies of tBid interactions with mitochondrial lipids using liposomes and the CHO-PGS-S cell line [75]. Wang and co-workers showed that the pro-apoptotic protein tBid interacts exclusively with liposomes that contain at least physiological levels of $\mathrm{CL}$ and demonstrated that tBid co-localization with CHO-PGS-S mitochondria is CL-dependent. The CL-binding domain of tBid was mapped to helices 4-6 of the Bid protein [75]. Interestingly, helix 6 was later shown to be a part of a hairpin structure which is important for the lipid binding properties of tBid [76]. Subsequently, electron tomogram studies showed that tBid interacts with mitochondria specifically at the inner and outer membrane contact sites, which are rich in CL (Fig. 4(A))
[77]. As discussed above, CL-rich membranes may adopt a non-bilayer hexagonal $\mathrm{H}_{\text {II }}$ configuration at the contact sites (Fig. 2), enabling access of CL to the cytosolic surface of mitochondria [27]. The model of CL as a mitochondrial "docking" site for tBid is supported by several studies. For example, in vitro assays using artificial membranes or isolated mitochondria showed that recombinant tBid can bind CL and MLCL [78-83]. Adding tBid to isolated mitochondria immediately inhibits ADP-stimulated respiration and oxidative phosphorylation, as a result of ANT inactivation $[84,85]$. The function of tBid may be either BH3-domain-dependent or independent. The former induces oligomerization of the multi-BH domain pro-apoptotic $\mathrm{Bcl}-2$ proteins $\mathrm{Bax}$ and $\mathrm{Bak}$ on the mitochondrial outer membrane, 
while a BH3-independent interaction of tBid with CL [82] could be responsible for cristae remodelling, [80] and for inhibition of oxidative phosphorylation [85]. Cristae remodelling and perturbations of mitochondrial bioenergetics take place simultaneously and are both independent of Bak. It is possible therefore that $\mathrm{tBid}$ acts by two sequential mechanisms: the first is $\mathrm{BH} 3$ domain independent, which involves CL, leading to structural and functional impairment, and the second is $\mathrm{BH} 3$ domain dependent, employing interactions with other pro-apoptotic $\mathrm{Bcl}-2$ proteins, namely Bak and Bax, leading to mitochondrial outer membrane permeabilization. Thus, the interaction of tBid with CL may prime mitochondria for the action of Bax and Bak.

\section{Cardiolipin redistribution}

Another feature of CL observed under apoptotic conditions is its redistribution within and between membranes. The exposure of $C L$ on the outer leaflet of the mitochondrial inner membrane was observed after death receptor stimulation before mitochondria depolarization and PS exposure on the plasma membrane, and at the same time as ROS generation [86]. Peroxidation of CL may account for their redistribution by altering their molecular organization and favouring formation of a non-bilayer hexagonal structure [87]. This could increase exposure of $\mathrm{CL}$ on the contact sites of mitochondrial membranes and provide access for tBid. It is also suggested that Bid, which exhibits lipid transfer activity in vitro, relocates CL and MLCL to the plasma membrane of cells undergoing apoptosis $[88,89]$. The mechanism and the significance of this relocation are still unclear. In addition, tBid may reorganize CL into micro-domains as was demonstrated in artificial lipid monolayers containing physiological amount of CL [85]. Considering the possible role of CL in maintaining mitochondrial structure, changes in CL organization may result in structural changes of the mitochondrial inner membrane which in turn may affect the activity of membraneembedded proteins such as ANT. Therefore, it is conceivable that $\mathrm{tBid}$ affects the structure and function of mitochondria by binding to and redistributing mitochondrial $\mathrm{CL}$ within the mitochondrial inner membrane and/or within other cellular compartments.

Cardiolipin and permeabilization of the mitochondrial outer membrane

CL was also proposed to be required for the action of other pro-apoptotic Bcl-2 proteins [90]. To study individual functions of Bcl-2-family proteins Newmeyer and co-workers took an in vitro approach using liposomes and outer mitochondrial membrane vesicles. Their work has provided evidence that permeabilization of liposomes to dextran required both the presence of activated Bax and physiological levels of CL. Therefore, it was suggested that Bax may permeabilize the mitochondrial outer membrane by altering the local organization of $\mathrm{CL}$ without overall damage to the membrane itself (Fig. 4(B)). In contrast to this report, other studies using either the CL synthase deficient yeast $\operatorname{crdl} \Delta$, or mitochondria from CL synthase knocked-down cells have shown that Bax does not require CL for the induction of cytochrome $c$ release [52, 84, 91]. However, as discussed above, it is possible that in yeast, PG, which accumulates in the absence of CL synthase, compensates for the loss of CL. It still awaits clarification whether $\mathrm{CL}$ or its PG precursor are needed, for Bax to release cytochrome $c$ [92]. This is particularly interesting since hydrophobic and electrostatic interactions make a different contribution to the binding of cytochrome $c$ to $\mathrm{CL}$ or PG [93].

\section{Cardiolipin-cytochrome $c$ peroxidase activity}

CL peroxidation appears to be an early event preceding the release of cytochrome $c$ and caspase activation. The mechanism of CL peroxidation and its involvement in apoptosis has gained more attention recently [58, 94]. Kagan and colleagues showed that cytochrome $c$ can interact with CL that contains two or more unsaturated acyl groups $\left(\mathrm{C}_{18: 2}\right.$ mostly) to form a hydrogen peroxide peroxidase capable of oxidizing CL to peroxi-CL (Fig. 3). Using cytochrome $c^{-/-}$mouse embryonic cells they provided the first evidence that cytochrome $c$ is required for the peroxidation of CL. CL-cytochrome $c$ complex acts as a potent CL-specific oxygenase required for the release of pro-apoptotic factors such as cytochrome $c$ and smac/diablo. It is noteworthy that oxidized CL does not merely allow cytochrome $c$ to detach from the mitochondrial inner membrane but rather has an active role in inducing apoptosis: when added to isolated mitochondria, oxidized CL alone induces cytochrome $c$ and smac/diablo release [58]. Importantly, the peroxidase activity of the CL-cytochrome $c$ complex depends on unsaturated acyl chains on CL. Indeed, incubation of HL60 cells with the poly-unsaturated fatty acid docosahexaenoic acid $\left(\mathrm{C}_{22: 6}\right)$, enriches $\mathrm{CL}$ with $\mathrm{C}_{22: 6}$ acyl chains, sensitizing the cells to staurosporine-induced apoptosis [58]. This promoted the notion that enriching CL with saturated acyl chains may protect from apoptosis. But although in vitro, saturated CL cannot stimulate CL-cytochrome $c$ peroxidase activity [58], CL synthase in a cellular context does not incorporate saturated PG to form fully saturated CL [62]. Nevertheless, the results, together with the suggestion that oxidized CL may have a promoting effect on the pro-apoptotic activity of Bcl-2 proteins, point to the importance of CL acyl chain composition and suggest that manipulation of CL oxidation may present a good target for sensitising cells to apoptosis. This also raises the question whether Bcl-2 proteins can regulate CL-cytochrome $c$ peroxidase activity. 


\section{Conclusions and perspectives}

Since their characterization in 1942 research has yielded increasing knowledge of the structure, localization and biosynthetic pathway of CL. Due to its specific location in the mitochondrial membranes, $\mathrm{CL}$ has long been viewed through its interactions with mitochondrial proteins: because it is required for the optimal activity of most of the respiratory chain complexes and of several mitochondrial substrate carriers, CL is crucial for efficient oxidative phosphorylation and for correct function and structure of the mitochondrial inner membrane.

In addition to its role in maintaining mitochondrial integrity, it is now clear that CL participates in the mitochondrial apoptotic pathway. CL is turning out to be involved in many of the mitochondrial-dependent steps that lead to the release of apoptogenic factors. These steps include interactions with Bcl-2 proteins, cytochrome $c$ association with and dissociation from the mitochondrial inner membrane, alteration of the structure of the mitochondrial inner membrane and permeabilization of the outer one. Moreover, this review emphasized that CL undergoes both reorganisation and modification within the mitochondrial membranes. Degradation of CL into MLCL and transition of CL to the mitochondrial outer membrane (and potentially to the plasma membrane) appear to be early events in apoptosis. Peroxidation of the acyl chains of CL also plays a crucial role in its apoptotic functions. In fact, this event is catalyzed by cytochrome $c$ and is required for the release of cytochrome $c$ itself, and of other apoptogenic factors, from mitochondria.

By way of analogy with sphingomyelin in the plasma membrane, it is conceivable that CL participates in the formation of signalling platforms in the mitochondrial membranes. The arrival of an apoptotic stimulus at the mitochondrial surface may result in the redistribution of CL into microdomains and in further amplification of the apoptotic signal. This reorganization of CL could lead to the remodelling of the mitochondrial cristae and to the loss of mitochondrial functions observed during apoptosis. However, the presence of CL domains in mitochondria, their structure and the effect of peroxidation on their organization are not well understood.

This review also highlighted the importance of CL acyl chain composition. CL strictly contains unsaturated fatty acyl chains, which are readily oxidizable targets. Manipulating the degree of saturation of the CL acyl chains may represent a new means of controlling the cell's fate. New strategies designed to pharmacologically manipulate the oxidation sensitivity of CL may help control cell death and open new prospects for the treatment of pathologies with diminished or excessive apoptosis. The recent surge of research into CL will hopefully provide better understanding both of acyl chain remodelling and of the role of CL in apoptosis in the near future.

\section{References}

1. Danial NN, Korsmeyer SJ (2004) Cell death: critical control points. Cell 116:205-219

2. Newmeyer DD, Ferguson-Miller S (2003) Mitochondria: releasing power for life and unleashing the machineries of death. Cell $112: 481-490$

3. Wang X (2001) The expanding role of mitochondria in apoptosis. Genes Dev 15:2922-2933

4. Kuida K, Haydar TF, Kuan CK et al (1998) Reduced apoptosis and cytochrome c-mediated caspase activation in mice lacking caspase 9. Cell 94:325-337

5. Kuida K, Zheng TS, Na S et al (1996) Decreased apoptosis in the brain and premature lethality in CPP32-deficient mice. Nature 384:368-372

6. Li K, Li Y, Shelton JM et al (2000) Cytochrome $c$ deficiency causes embryonic lethality and attenuates stress-induced apoptosis. Cell 101:389-399

7. Yoshida H, Kong YY, Yoshida R et al (1998) Apaf1 is required for mitochondrial pathways of apoptosis and brain development. Cell 94:739-750

8. Garrido C, Galluzzi L, Brunet M et al (2006) Mechanisms of cytochrome $c$ release from mitochondria. Cell Death Differ 13:1423-1433

9. van Loo G, Saelens X, van Gurp M et al (2002) The role of mitochondrial factors in apoptosis: a Russian roulette with more than one bullet. Cell Death Differ 9:1031-1042

10. Fadok VA, Voelker DR, Campbell PA et al (1992) Exposure of phophatidylserine on the surface of apoptotic lymphocytes triggers specific recognition and removal by macrophages. J Immunol 148:2207-2216

11. Siskind LJ (2005) Mitochondrial ceramide and the induction of apoptosis. J Bioenerg Biomembr 37:143-153

12. Hauff KD, Hatch GM (2006) Cardiolipin metabolism and Barth Syndrome. Prog Lipid Res 45:91-101

13. Schlame M, Rua D, Greenberg ML (2000) The biosynthesis and functional role of cardiolipin. Prog Lipid Res 39:257-288

14. Houtkooper RH, Akbari H, van Lenthe $H$ et al (2006) Identification and characterization of human cardiolipin synthase. FEBS Lett 580:3059-3064

15. Hoch FL (1992) Cardiolipins and biomembrane function. Biochim Biophys Acta 1113:71-133

16. Schlame M, Ren M, Xu Y, Greenberg ML, Haller I (2005) Molecular symmetry in mitochondrial cardiolipins. Chem Phys Lipids 138:38-49

17. Rustow B, Schlame M, Rabe H, Reichmann G, Kunze D (1989) Species pattern of phosphatidic acid, diacylglycerol, CDPdiacylglycerol and phosphatidylglycerol synthesized de novo in rat liver mitochondria. Biochim Biophys Acta 1002:261-263

18. Schlame M, Rustow B (1990) Lysocardiolipin formation and reacylation in isolated rat liver mitochondria. Biochem J 272:589-595

19. Xu Y, Kelley RI, Blanck TJ, Schlame M (2003) Remodeling of cardiolipin by phospholipid transacylation. J Biol Chem 278:51380-51385

20. Barth PG, Scholte HR, Berden JA et al (1983) An X-linked mitochondrial disease affecting cardiac muscle, skeletal muscle and neutrophil leucocytes. J Neurol Sci 62:327-355

21. Kelley RI, Cheatham JP, Clark BJ et al (1991) X-linked dilated cardiomyopathy with neutropenia, growth retardation, and 3-methylglutaconic aciduria. J Pediatr 119:738-747

22. Bione S, D'Adamo P, Maestrini E et al (1996) A novel X-linked gene, G4.5. is responsible for Barth syndrome. Nat Genet 12:385-389

23. Neuwald AF (1997) Barth syndrome may be due to an acyltransferase deficiency. Curr Biol 7:R465-R466 
24. Schlame M, Kelley RI, Feigenbaum A et al (2003) Phospholipid abnormalities in children with Barth syndrome. J Am Coll Cardiol 42:1994-1999

25. Valianpour F, Mitsakos V, Schlemmer D et al (2005) Monolysocardiolipins accumulate in Barth syndrome but do not lead to enhanced apoptosis. J Lipid Res 46:1182-1195

26. Krebs JJ, Hauser H, Carafoli E (1979) Asymmetric distribution of phospholipids in the inner membrane of beef heart mitochondria. J Biol Chem 254:5308-5316

27. Ardail D, Privat JP, Egret-Charlier M et al (1990) Mitochondrial contact sites. Lipid composition and dynamics. J Biol Chem 265:18797-18802

28. de Kroon AI, Dolis D, Mayer A, Lill R, de Kruijff B (1997) Phospholipid composition of highly purified mitochondrial outer membranes of rat liver and Neurospora crassa. Is cardiolipin present in the mitochondrial outer membrane? Biochim Biophys Acta 1325:108-116

29. Hovius R, Lambrechts H, Nicolay K, de Kruijff B (1990) Improved methods to isolate and subfractionate rat liver mitochondria. Lipid composition of the inner and outer membrane. Biochim Biophys Acta 1021:217-226

30. Cullis PR, Verkleij AJ, Ververgaert PH (1978) Polymorphic phase behaviour of cardiolipin as detected by 31P NMR and freeze-fracture techniques. Effects of calcium, dibucaine and chlorpromazine. Biochim Biophys Acta 513:11-20

31. Van Venetie R, Verkleij AJ (1982) Possible role of non-bilayer lipids in the structure of mitochondria. A freeze-fracture electron microscopy study. Biochim Biophys Acta 692:397-405

32. Eble KS, Coleman WB, Hantgan RR, Cunningham CC (1990) Tightly associated cardiolipin in the bovine heart mitochondrial ATP synthase as analyzed by 31P nuclear magnetic resonance spectroscopy. J Biol Chem 265:19434-19440

33. Fry M, Green DE (1981) Cardiolipin requirement for electron transfer in complex I and III of the mitochondrial respiratory chain. J Biol Chem 256:1874-1880

34. Sedlak E, Panda M, Dale MP, Weintraub ST, Robinson NC (2006) Photolabeling of cardiolipin binding subunits within bovine heart cytochrome $c$ oxidase. Biochemistry 45:746-754

35. Yue WH, Zou YP, Yu L, Yu CA (1991) Crystallization of mitochondrial ubiquinol-cytochrome $c$ reductase. Biochemistry 30:2303-2306

36. Yankovskaya V, Horsefield R, Tornroth S et al (2003) Architecture of succinate dehydrogenase and reactive oxygen species generation. Science 299:700-704

37. Bisaccia F, Palmieri F (1984) Specific elution from hydroxylapatite of the mitochondrial phosphate carrier by cardiolipin. Biochim Biophys Acta 766:386-394

38. Hoffmann B, Stockl A, Schlame M, Beyer K, Klingenberg M (1994) The reconstituted ADP/ATP carrier activity has an absolute requirement for cardiolipin as shown in cysteine mutants. J Biol Chem 269:1940-1944

39. Nalecz KA, Bolli R, Wojtczak L, Azzi A (1986) The monocarboxylate carrier from bovine heart mitochondria: partial purification and its substrate-transporting properties in a reconstituted system. Biochim Biophys Acta 851:29-37

40. Noel H, Pande SV (1986) An essential requirement of cardiolipin for mitochondrial carnitine acylcarnitine translocase activity. Lipid requirement of carnitine acylcarnitine translocase. Eur J Biochem 155:99-102

41. Ohtsuka T, Nishijima M, Akamatsu Y (1993) A somatic cell mutant defective in phosphatidylglycerophosphate synthase, with impaired phosphatidylglycerol and cardiolipin biosynthesis. J Biol Chem 268:22908-22913

42. Ohtsuka T, Nishijima M, Suzuki K, Akamatsu Y (1993) Mitochondrial dysfunction of a cultured Chinese hamster ovary cell mutant deficient in cardiolipin. J Biol Chem 268:22914-22919
43. Jiang F, Rizavi HS, Greenberg ML (1997) Cardiolipin is not essential for the growth of Saccharomyces cerevisiae on fermentable or non-fermentable carbon sources. Mol Microbiol 26:481-491

44. Jiang F, Ryan MT, Schlame M et al (2000) Absence of cardiolipin in the crd1 null mutant results in decreased mitochondrial membrane potential and reduced mitochondrial function. J Biol Chem 275:22387-22394

45. Koshkin V, Greenberg ML (2000) Oxidative phosphorylation in cardiolipin-lacking yeast mitochondria. Biochem J 347(Pt 3):687691

46. Koshkin V, Greenberg ML (2002) Cardiolipin prevents ratedependent uncoupling and provides osmotic stability in yeast mitochondria. Biochem J 364:317-322

47. Bernardi P, Azzone GF (1981) Cytochrome $c$ as an electron shuttle between the outer and inner mitochondrial membranes. J Biol Chem 256:7187-7192

48. Scorrano L, Ashiya M, Buttle K et al (2002) A distinct pathway remodels mitochondrial cristae and mobilizes cytochrome $c$ during apoptosis. Dev Cell 2:55-67

49. Nicholls P (1974) Cytochrome $c$ binding to enzymes and membranes. Biochim Biophys Acta 346:261-310

50. Rytomaa M, Mustonen P, Kinnunen PK (1992) Reversible, nonionic, and $\mathrm{pH}$-dependent association of cytochrome $c$ with cardiolipin-phosphatidylcholine liposomes. J Biol Chem 267:22243-22248

51. Kawasaki K, Kuge O, Chang SC et al (1999) Isolation of a chinese hamster ovary (CHO) cDNA encoding phosphatidylglycerophosphate (PGP) synthase, expression of which corrects the mitochondrial abnormalities of a PGP synthase-defective mutant of CHO-K1 cells. J Biol Chem 274:1828-1834

52. Choi SY, Gonzalvez F, Jenkins GM et al (2006) Cardiolipin deficiency releases cytochrome $c$ from the inner mitochondrial membrane and accelerates stimuli-elicited apoptosis. Cell Death Differ advance online publication, doi:10.1038/sj.cdd.4402020

53. Chang SC, Heacock PN, Mileykovskaya E, Voelker DR, Dowhan W (1998) Isolation and characterization of the gene (CLS1) encoding cardiolipin synthase in Saccharomyces cerevisiae. J Biol Chem 273:14933-14941

54. Paradies G, Petrosillo G, Pistolese M et al (1999) Lipid peroxidation and alterations to oxidative metabolism in mitochondria isolated from rat heart subjected to ischemia and reperfusion. Free Radic Biol Med 27:42-50

55. Paradies G, Ruggiero FM, Petrosillo G, Quagliariello E (1997) Age-dependent decline in the cytochrome $c$ oxidase activity in rat heart mitochondria: role of cardiolipin. FEBS Lett 406:136138

56. Paradies G, Petrosillo G, Pistolese M, Ruggiero FM (2000) The effect of reactive oxygen species generated from the mitochondrial electron transport chain on the cytochrome $c$ oxidase activity and on the cardiolipin content in bovine heart submitochondrial particles. FEBS Lett 466:323-326

57. Paradies G, Petrosillo G, Pistolese M, Ruggiero FM (2002) Reactive oxygen species affect mitochondrial electron transport complex I activity through oxidative cardiolipin damage. Gene 286:135-141

58. Kagan VE, Tyurin VA, Jiang J et al (2005) Cytochrome $c$ acts as a cardiolipin oxygenase required for release of proapoptotic factors. Nat Chem Biol 1:223-232

59. Kirkland RA, Adibhatla RM, Hatcher JF, Franklin JL (2002) Loss of cardiolipin and mitochondria during programmed neuronal death: evidence of a role for lipid peroxidation and autophagy. Neuroscience 115:587-602

60. Ushmorov A, Ratter F, Lehmann V et al (1999) Nitric-oxideinduced apoptosis in human leukemic lines requires mitochondrial lipid degradation and cytochrome $c$ release. Blood 93:23422352 
61. Hardy S, El-Assaad W, Przybytkowski E et al (2003) Saturated fatty acid-induced apoptosis in MDA-MB-231 breast cancer cells. A role for cardiolipin. J Biol Chem 278:31861-31870

62. Ostrander DB, Sparagna GC, Amoscato AA, McMillin JB, Dowhan W (2001) Decreased cardiolipin synthesis corresponds with cytochrome $c$ release in palmitate-induced cardiomyocyte apoptosis. J Biol Chem 276:38061-38067

63. Rytomaa M, Kinnunen PK (1995) Reversibility of the binding of cytochrome $c$ to liposomes. Implications for lipid-protein interactions. J Biol Chem 270:3197-3202

64. Gorbenko GP (1999) Structure of cytochrome $c$ complexes with phospholipids as revealed by resonance energy transfer. Biochim Biophys Acta 1420:1-13

65. Cortese JD, Voglino AL, Hackenbrock CR (1995) Persistence of cytochrome $c$ binding to membranes at physiological mitochondrial intermembrane space ionic strength. Biochim Biophys Acta 1228:216-228

66. Pereverzev MO, Vygodina TV, Konstantinov AA, Skulachev VP (2003) Cytochrome $c$, an ideal antioxidant. Biochem Soc Trans 31:1312-1315

67. Nomura K, Imai H, Koumura T, Kobayashi T, Nakagawa Y (2000) Mitochondrial phospholipid hydroperoxide glutathione peroxidase inhibits the release of cytochrome $c$ from mitochondria by suppressing the peroxidation of cardiolipin in hypoglycaemia-induced apoptosis. Biochem J 351:183-193

68. Shidoji Y, Hayashi K, Komura S, Ohishi N, Yagi K (1999) Loss of molecular interaction between cytochrome $c$ and cardiolipin due to lipid peroxidation. Biochem Biophys Res Commun 264:343-347

69. Ott M, Robertson JD, Gogvadze V, Zhivotovsky B, Orrenius S (2002) Cytochrome $c$ release from mitochondria proceeds by a two-step process. Proc Natl Acad Sci USA 99:12591263

70. Gottlieb E (2006) OPA1 and PARL keep a lid on apoptosis. Cell $126: 27-29$

71. Adams JM, Cory S (1998) The Bcl-2 protein family: arbiters of cell survival. Science 281:1322-1326

72. Li H, Zhu H, Xu CJ, Yuan J (1998) Cleavage of BID by caspase 8 mediates the mitochondrial damage in the Fas pathway of apoptosis. Cell 94:491-501

73. Luo X, Budihardjo I, Zou H, Slaughter C, Wang X (1998) Bid, a bcl-2 interacting protein, mediates cytochrome $c$ release from mitochondria in response to activation of cell surface receptors. Cell 94:481-490.

74. Cartron PF, Gallenne T, Bougras G et al (2004) The first alpha helix of Bax plays a necessary role in its ligand-induced activation by the BH3-only proteins Bid and PUMA. Mol Cell 16:807818

75. Lutter M, Fang M, Luo X et al (2000) Cardiolipin provides specificity for targeting of tBid to mitochondria. Nat Cell Biol 2:754-761

76. Garcia-Saez AJ, Mingarro I, Perez-Paya E, Salgado J (2004) Membrane-insertion fragments of Bcl-xL, Bax, and Bid. Biochemistry 43:10930-10943

77. Lutter M, Perkins GA, Wang X (2001) The pro-apoptotic Bcl-2 family member tBid localizes to mitochondrial contact sites. BMC Cell Biol 2:22
78. Epand RF, Martinou JC, Fornallaz-Mulhauser M, Hughes DW, Epand RM (2002) The apoptotic protein tBid promotes leakage by altering membrane curvature. J Biol Chem 277:32632-32639

79. Esposti MD, Cristea IM, Gaskell SJ, Nakao Y, Dive $C$ (2003) Proapoptotic bid binds to monolysocardiolipin, a new molecular connection between mitochondrial membranes and cell death. Cell Death Differ 10:1300-1309

80. Kim TH, Zhao Y, Ding WX et al (2004) Bid-cardiolipin interaction at mitochondrial contact site contributes to mitochondrial cristae reorganization and cytochrome $c$ release. Mol Biol Cell 15:3061-3072

81. Liu J, Durrant D, Yang HS et al (2005) The interaction between tBid and cardiolipin or monolysocardiolipin. Biochem Biophys Res Commun 330:865-870

82. Liu J, Weiss A, Durrant D, Chi NW, Lee RM (2004) The cardiolipin-binding domain of Bid affects mitochondrial respiration and enhances cytochrome $c$ release. Apoptosis 9:533-541

83. Zha J, Weiler S, Oh KJ, Wei MC, Korsmeyer SJ (2000) Posttranslational $\mathrm{N}$-myristoylation of BID as a molecular switch for targeting mitochondria and apoptosis. Science 290:1761-1765

84. Gonzalvez F, Bessoule JJ, Rocchiccioli F, Manon S, Petit PX (2005) Role of cardiolipin on tBid and tBid/Bax synergistic effects on yeast mitochondria. Cell Death Differ 12:659-667

85. Gonzalvez F, Pariselli F, Dupaigne P et al (2005) tBid interaction with cardiolipin primarily orchestrates mitochondrial dysfunctions and subsequently activates Bax and Bak. Cell Death Differ $12: 614-626$

86. Garcia Fernandez M, Troiano L, Moretti L et al (2002) Early changes in intramitochondrial cardiolipin distribution during apoptosis. Cell Growth Differ 13:449-455

87. Aguilar L, Ortega-Pierres G, Campos B et al (1999) Phospholipid membranes form specific nonbilayer molecular arrangements that are antigenic. J Biol Chem 274:25193-25196

88. Esposti MD, Erler JT, Hickman JA, Dive $c$ (2001) Bid, a widely expressed proapoptotic protein of the Bcl-2 family, displays lipid transfer activity. Mol Cell Biol 21:7268-7276

89. Sorice M, Circella A, Cristea IM et al (2004) Cardiolipin and its metabolites move from mitochondria to other cellular membranes during death receptor-mediated apoptosis. Cell Death Differ 11:1133-1145

90. Kuwana T, Mackey MR, Perkins G et al (2002) Bid, bax, and lipids cooperate to form supramolecular openings in the outer mitochondrial membrane. Cell 111:331-342

91. Iverson SL, Enoksson M, Gogvadze V, Ott M, Orrenius S (2004) Cardiolipin is not required for Bax-mediated cytochrome $c$ release from yeast mitochondria. J Biol Chem 279:1100-1107

92. Polcic P, Su X, Fowlkes J et al (2005) Cardiolipin and phosphatidylglycerol are not required for the in vivo action of Bcl-2 family proteins. Cell Death Differ 12:310-312

93. Rytomaa M, Kinnunen PK (1994) Evidence for two distinct acidic phospholipid-binding sites in cytochrome $c$. J Biol Chem 269:1770-1774

94. Kagan VE, Tyurina YY, Bayir H et al (2006) The "pro-apoptotic genies" get out of mitochondria: oxidative lipidomics and redox activity of cytochrome $c /$ cardiolipin complexes. Chem Biol Interact 163:15-28 